\title{
The DICA Endoscopic Classification for Diverticular Disease of the Colon Shows a Significant Interobserver Agreement among Community Endoscopists: an International Study
}

\begin{abstract}
Antonio Tursi ${ }^{1}$, Giovanni Brandimarte ${ }^{2}$, Francesco Di Mario ${ }^{3}$, Angel Lanas ${ }^{4}$, Carmelo Scarpignato ${ }^{5}$, Mauro Bafutto ${ }^{6}$, Giovanni Barbara $^{7}$, Gabrio Bassotti ${ }^{8}$, Gian Andrea Binda ${ }^{9}$, Alberto Biondi ${ }^{10}$, Sebastiano Biondo ${ }^{11}$, Claudio Cassieri ${ }^{2}$, Antonio Crucitti ${ }^{12}$, Dan L. Dumitrașcu ${ }^{13}$, Walter Elisei ${ }^{14}$, Ricardo Escalante ${ }^{15}$, László Herszenyi ${ }^{16}$, Wolfgang Kruis ${ }^{17}$, Juozas Kupcinskas $^{18}$, Adi Lahat ${ }^{19}$, Piera G. Lecca ${ }^{2}$, Giovanni Maconi ${ }^{20}$, Peter Malfertheiner ${ }^{21}$, Andrea Mazzarri ${ }^{12}$, Fermìn Mearin ${ }^{22}$, Tomica Milosavljevic ${ }^{23}$, Gerardo Nardone ${ }^{24}$, Enio Chavez de Oliveira ${ }^{25}$, Alfredo Papa ${ }^{26}$, Savvas Papagrigoriadis ${ }^{27}$, Miguel Pera $^{28}$, Roberto Persiani ${ }^{10}$, Marcello Picchio ${ }^{29}$, Jaroslaw Regula ${ }^{30}$, Davor Štimac ${ }^{31}$, Neil Stollman ${ }^{32}$, Lisa L. Strate ${ }^{33}$, Marjorie M.D. Walker ${ }^{34}$, and DICA International Group (see Appendix)
\end{abstract}

1) Gastroenterology Service, ASL BAT, Andria (BT), Italy; 2) Division of Internal Medicine and Gastroenterology, "Cristo Re" Hospital, Rome, Italy; 3) Department of Medicine and Surgery, Gastroenterology Unit, University of Parma, Parma, Italy; 4) Service of Digestive Diseases, University Clinic Hospital Lozano Blesa, University of Zaragoza, IIS Aragón, Zaragoza, Spain; 5) Faculty of Medical Sciences, United Campus of Malta, San Giljan, Malta ; 6) Consultório, R, 46 - 25 Clin de Gastroentero (St Coimbra) Goiânia, Goiás (GO), Brazil; 7) Department of Clinical Medicine, Section of Gastroenterology, "S. Orsola” Hospital, University of Bologna, Bologna, Italy; 8) Department of Clinical and Experimental Medicine, Gastroenterology and Hepatology Section, "Santa Maria della Misericordia" Hospital, University of Perugia, San Sisto (PG), Italy; 9) Department of Surgery, "Galliera” Hospital, Genoa, Italy; 10) Department of Surgical Sciences, First General Surgery Unit, Catholic University - „A. Gemelli” University Hospital, Rome, Italy; 11) Department of General and Digestive Surgery, Colorectal Unit, Bellvitge University Hospital and IDIBELL, University of Barcelona, Barcelona, Spain; 12) Division of General Surgery, „Cristo Re” Hospital, Rome, Italy; 13) 2nd Medical Department, Iuliu Hatieganu University of Medicine and Pharmacy Cluj-Napoca, Romania; 14) Division of Gastroenterology, ASL Roma 6, Albano Laziale, Rome, Italy; 15) Universidad Central de Venezuela, Loira Medical Center, Caracas, Venezuela; 16) 2nd Department of Medicine, Semmelweis University, H-1088 Budapest, Hungary; 17) Innere Medizin, Evangelische Krankenhaus Kalk, Cologne, Germany; 18) Department of Gastroenterology, Lithuanian University of Health Sciences, Kaunas, Lithuania; 19) Department of Gastroenterology, Chaim Sheba Medical Center, Tel Hashomer, Ramat Gan 52651, Israel; 20) Gastrointestinal Unit, Department of Clinical Sciences, "L.Sacco" University Hospital, Milan, Italy; 21) Department of Gastroenterology, Hepatology and Infectious Diseases, Otto-vonGuericke University Hospital, Magdeburg, Germany; 22) Servicio Aparato Digestivo, Centro Médico Teknon, Barcelona, Spain; 23) Clinical Centre of Serbia, University of Belgrade, Serbia; 24) Department of Clinical and Experimental Medicine, University „Federico II”, Naples, Italy; 25) Av, 470 - Hosp. Ort. de Goiania (St Aeroporto), Goiânia, Goiás (GO), Brasil; 26) Division of Internal Medicine and Gastroenterology, CIC "Columbus", Catholic University - “A. Gemelli” University Hospital, Rome, Italy; 27) Department of Colorectal Surgery, King's College Hospital, London, United Kingdom; 28) Sección de Cirugía Gastrointestinal, Hospital Universitari del Mar, Barcelona, Spain; 29) Division of General Surgery, "P. Colombo" Hospital, ASL Roma 6, Velletri, Roma, Italy; 30) Department of Gastroenterology, Hepatology and Clinical Oncology, Medical Postgraduate Education Centre; Department of Gastrointestinal Oncology, Maria Sklodowska-Curie Clinical Oncology Institute, Warsaw, Poland; 31) Division of Internal Medicine, University Hospital, Rijeka, Croatia; 32) Division of Gastroenterology, Department of Medicine, University of California San Francisco, Oakland, CA, USA; 33) Division of Gastroenterology, Department of Medicine, Harborview Medical Center, University of Washington Medical School, Seattle, Washington, USA; 34) University of Newcastle, Faculty of Health and Medicine, School of Medicine \& Public Health, Australian Gastrointestinal Research Alliance, Callaghan NSW 2308, Australia

\footnotetext{
Address for correspondence: Antonio Tursi, MD Servizio di Gastroenterologia Territoriale DSS $n^{\circ} 4$, ASL BAT Via Torino, 49 76123 Andria (BT), Italy antotursi@tiscali.it
}

\begin{abstract}
Background \& Aims: The Diverticular Inflammation and Complication Assessment (DICA) endoscopic classification of diverticulosis and diverticular disease (DD) is currently available. It scores severity of the disease as DICA 1, DICA 2 and DICA 3. Our aim was to assess the agreement on this classification in an international endoscopists community setting.

Methods: A total of 96 doctors (82.9\% endoscopists) independently scored a set of DD endoscopic videos. The percentages of overall agreement on DICA score and a free-marginal multirater kappa $(\kappa)$ coefficient were reported as statistical measures of interrater agreement.
\end{abstract}


Results: Overall agreement in using DICA was $91.8 \%$ with a free-marginal kappa of $88 \%$ (95\% CI 80-95). The overall agreement levels were: DICA 1, 85.2\%; DICA 2, 96.5\%; DICA 3, 99.5\%. The free marginal $\kappa$ was: DICA $1=0.753$, DICA $2=0.958$, DICA $3=0.919$. The agreement about the main endoscopic items was $83.4 \%(\mathrm{k}$ $67 \%$ ) for diverticular extension, $62.6 \%(\mathrm{k} 65 \%)$ for number of diverticula for each district, $86.8 \%(\mathrm{k} 82 \%)$ for presence of inflammation, and 98.5 (k 98\%) for presence of complications.

Conclusions: The overall interrater agreement in this study ranges from good to very good. DICA score is a simple and reproducible endoscopic scoring system for diverticulosis and DD.

Key words: agreement - colonoscopy - community setting - diverticular disease of the colon- endoscopic classification.

Abbreviations: AD: acute diverticulitis; CT: computer tomography; DD: diverticular disease; DICA: Diverticula Inflammation and Complications Assessment; SCAD: segmental colitis associated with diverticulosis.

\section{INTRODUCTION}

The most frequent anatomic alteration detected during colonoscopy is the colonic diverticulosis [1]. The prevalence of diverticulosis increases with age. However, it remains generally asymptomatic, and only about $20-25 \%$ of those people may develop symptoms that can be linked to diverticulosis, developing the so-called "Diverticular Disease" (DD) [2]. As many diseases, DD may be scored in several ways. Imaging classifications, assessing particularly the appearance of the disease by abdominal computer tomography (CT), are the most used [3-5]. Other classifications focused their attention on the clinical appearance of the disease, trying to identify patients with different risks of severity [6-8]. However, most of them focused their attention on the severity of the acute diverticulitis (AD) rather than on the overall spectrum of the disease.

Although the large number of colonoscopies currently performed in real life, and the frequent detection of endoscopic signs of diverticular inflammation in patients submitted to colonoscopy $[9,10]$, an endoscopic classification of the disease was lacking until 2015. In that year, the first endoscopic classification of DD, called "DICA" (Diverticula Inflammation and Complications Assessment), has been developed and validated [11]. This classification takes into consideration several scored items (extension of diverticulosis, number of diverticula for each district, presence of inflammatory signs and occurrence of complications) and the relative sub-items, the sum of them leading to three different DICA scores (DICA 1, DICA 2, and DICA 3). The importance of this classification in linked to its predictive value. An international, retrospective study recently found this classification effective in predicting the course of the disease in terms of $\mathrm{AD}$ occurrence/recurrence and surgery occurrence [12], and the preliminary data from a prospective study seem to confirm the prior results [13].

Some criticisms have been raised because this classification has been developed and validated by expert endoscopists. However, a recent study conducted in Italy found a significant interobserver agreement also in real life [14]. The aim of the present study was to assess the agreement about DICA classification involving a large community of physicians coming from several countries.

\section{METHODS}

The reproducibility level of the DICA endoscopic classification was evaluated in an interobserver variation study conducted during the 33d International Symposium on Diverticular Disease, held in Madrid on April 11-13, 2019.

In order to select the videos, the promoters of DICA classification (A. Tursi, G. Brandimarte and F. Di Mario) retrospectively reviewed 100 videos coming from their centers and showing colonic diverticular disease. All videos were completely anonymous, and all patients gave written informed consent before undergoing colonoscopy. Among them, 10 videos were randomly selected according to complete endoscopic exploration of the colon and showed to the audience for the scoring.

All videos were classified according to DICA classification [11], considering the following items and sub-items:

a. Diverticulosis extension: left colon (up to splenic flexure); right colon (over the splenic flexure).

b. Number of diverticula (in each district): up to 15: grade I; > 15: grade II).

c. Presence of inflammation: Edema/Hyperemia; Erosions; Segmental colitis Associated with Diverticulosis (SCAD). When different grades of inflammation were detected at the same time and in the same district (in example, some diverticula showing hyperemia and some showing erosions), the most severe grade of inflammation was reported.

\section{d. Presence of complications:}

- Rigidity of the colon: scarce distension of the diverticular district to inflation, including mild stenosis in which the standard colonoscope could be passed through the narrowed lumen;

- Stenosis: not passing stenosis or narrowed lumen with elevated risk of perforation due to presence of some anatomical characteristics (in example, multiple diverticula at the splenic flexure);

- Pus: purulent material coming out from diverticular opening.

- Bleeding.

Videos were classified by A. Tursi and G. Brandimarte. Conflicting classification was resolved by a third part (F. Di Mario). 
Visualization of the videos and assessment of the endoscopic variables under examination were performed during a plenary session lasting four hours. Participants had ten minutes to assess and rate each video, and scoring was made by using an anonymous chart collection. At the end of the session, a discussion of the results was performed. The promoters of the study took part into discussion but did not interfere with the decisions of the group.

All participants to the Post-Graduated course on DICA classification, held during the 3rd International Symposium on Diverticular Disease of the colon, scored the videos in order to assess the reproducibility of DICA classification in an international clinical setting. Not all the participants involved were endoscopists or knew the DICA classification, and not all of them used it in their practice. Thus, we assessed if they were endoscopists or not, if they had knowledge or not about DICA classification, and if they used it in their practice.

\section{Statistical analysis}

Agreement between evaluations of endoscopic findings was assessed through kappa statistics (a coefficient of interobserver agreement over and above the agreement that would be expected to occur by chance alone). Kappa values in percentage range from negative (disagreement) to 100 (total agreement) a value of $0 \%$ indicates agreement equal to that expected by pure chance; values below $40 \%$ are classified as poor agreement, $41-60 \%$ as moderate agreement, $61-80 \%$ as good agreement, and values above $80 \%$ as very good agreement [15]. Randolph's free-marginal multirater kappa was used [16].

\section{RESULTS}

The study group assessing videos consisted of 96 doctors coming from Europe (Bulgaria, Croatia, Germany, Hungary, Italy, Romania, Poland, Portugal, Slovakia, Spain, The Netherland, Russia), Africa (Tunisia), America (Brazil, Mexico, United States, Venezuela), and Australia. Endoscopists were 63 (82.9\%). DICA classification was known by 62 (81.6\%) doctors and used routinely by 37 (48.7\%) doctors.

As stated, 10 video clips were evaluated and rated by participants (960 visualizations were therefore performed): 4 videos were classified as DICA 1, 4 as DICA 2 and 2 as DICA 3.

The overall agreement level for DICA classification was $91.8 \%$ with a free-marginal kappa of $88 \%$ (95\% CI 80-95). The overall agreement levels were: DICA 1, 85.2\%; DICA 2, $96.5 \%$; DICA 3, 99.5\%. The free marginal $\kappa$ was: DICA $1=$ 0.753 , DICA $2=0.958$, DICA $3=0.919$. The agreement about the main endoscopic items was $83.4 \%$ ( $\mathrm{k} 67 \%$ ) for diverticular extension, $62.6 \%$ (k 65\%) for number of diverticula for each district, $86.8 \%$ (k 82\%) for presence of inflammation, and 98.5 (98\%) for presence of complications.

Agreement with respect to the four main items included in DICA classification are reported in Table I. Globally, percentage of agreement was very good for each item considered: $83.4 \%$ (k 67\%) for diverticular extension, $62.6 \%$ (k 65\%) for number of diverticula for each district, $86.8 \%$ (k $82 \%$ ) for presence of inflammation, and $98.5 \%$ (98\%) for presence of complications.
Table I. The percentage of interobserver agreement of endoscopic items in DICA classification

\begin{tabular}{lccc}
\hline Item & $\begin{array}{c}\text { Overall } \\
\text { agreement }\end{array}$ & $\begin{array}{c}\text { Free marginal } \\
\text { Kappa }\end{array}$ & 95\%CI \\
\hline Diverticula extent & 83.4 & 67 & $40-93$ \\
Number of diverticula & 82.6 & 65 & $36-95$ \\
Presence of inflammation & 86.8 & 82 & $69-96$ \\
Presence of complications & 98.5 & 98 & $96-100$ \\
\hline
\end{tabular}

\section{DISCUSSION}

Diverticulosis and DD is quite common in the Western World, more in the USA than in Europe, and its prevalence increases worldwide, probably due to progressive adoption of western lifestyle [1]. Thus, it has a significant impact on the National Health Systems.

Although DD can be classified according to several radiological and clinical approaches [3-8], the vast majority of them failed to have an adequate validation and cannot be proposed as standard reference. For many years, an endoscopic classification of the disease was lacking. The absence of any such endoscopic classification was even more evident considering the high number of colonoscopies currently performed, and the significant prevalence of diverticular inflammation detected everyday by colonoscopy $[9,10]$.

The first endoscopic classification of diverticulosis and DD, called DICA, has been recently developed and validated $[11,12]$. This classification was the first attempt to overcome definitions that too vaguely describe the colon with diverticulosis (as example, "scattered" of "diffuse" or "severe" diverticulosis). This classification described the four main items that can be detected during an endoscopic exploration of colon harboring diverticulosis (namely diverticulosis extension, number of diverticula in each district, presence or not of inflammation, and presence or absence of complications). Moreover, it leads to a 3-step simple score, which is linked to the outcome of the disease $[11,12]$.

Some criticisms have been raised because this classification has been developed and validated by expert endoscopists [11]. This choice probably influenced the results of the validation process, in which the interrater agreement was $\mathrm{k} 0.878$ for DICA 1, k 0.765 for DICA 2, and k 0.891for DICA 3 [11].

In a recent study conducted among Italian endoscopists community, we tried to overcome this limit [14]. Since DICA classification is not used by all endoscopists, we involved two types of population, namely endoscopists experts and endoscopists not experts with using this classification. We found a good inter-rater agreement in using this classification: the free marginal $\mathrm{k}$ ranged for moderate to good and, as expected, it was higher among endoscopists experts with DICA classification (the free marginal $\kappa$ ranged from good to very good) [14]. This is a significant result, because agreement in using endoscopic classifications is not always satisfactory even in expert endoscopists. For example, the Savary-Miller scoring system for esophagitis revealed moderate agreement for the experienced group while the Los Angeles system shows a slight improvement but irrespective of the level of experience 
[17]. Considering the inflammatory bowel diseases scoring systems, the results were similar. A recent study found that agreement between experienced endoscopists was suboptimal for Mayo subscore, fair for Rutgeerts score, and good for Crohn's Endoscopic Index of Severity and Simple Endoscopic Score for Crohn's Disease scores [18]. But the most important strength coming from that study was that we defined as "expert" physicians who used DICA classification at least three months in his/her practice: this means that the learning curve to obtain expertise about this classification is very short.

The present study, conducted involving participants to the Post-Graduated course on DICA classification, held during the 3rd International Symposium on Diverticular Disease of the Colon, confirms the DICA classification is easy to learn and to use in real life. We found that the agreement for DICA classification was excellent, since the free marginal $\kappa$ for the overall classification was $88 \%$. This excellent result could be a consequence of the high rate of DICA knowers and users among the raters. On the other hand, we have to consider that this International Meeting was monothematic, and probably has attracted doctors with specific interest in this disease (DICA classification included).

Moreover, the strength of this study is that an international audience was involved for the first time in this process of agreement, while the previous real-life study was conducted in Italy (where the classification was developed and firstly validated).

\section{CONCLUSION}

This international study has confirmed once again that DICA classification is a reproducible and easy-to-use endoscopic scoring system for diverticulosis and DD of the colon.

\section{APPENDIX}

DICA International Group: Leonardo Allegretta ${ }^{1}$, Nadia Altavilla ${ }^{2}$, Pedro Amaro ${ }^{3}$, Maria Laura Annunziata ${ }^{4}$, Franz Barberio ${ }^{5}$, Giuseppe Basile $^{6}$, Giuliano Bedogni ${ }^{7}$, Valerio Belfiori ${ }^{8}$, Stefano Benvenuti ${ }^{9}$, Carmelo Bertolami ${ }^{10}$, Marco Bisello ${ }^{11}$, Mouna Boudarous El Dammak $^{12}$, Rosamaria Bozzi ${ }^{13}$, Marisa Buono ${ }^{4}$, Ginevra Cambie ${ }^{14}$, Erminio Capezzuto ${ }^{15}$, Carlo Casamassima ${ }^{16}$, Ani Chavoushian ${ }^{17}$, Riccardo Ciofani ${ }^{18}$, Cinzia Citarella ${ }^{19}$, Debora Compare ${ }^{20}$, Bogdan Cotruta $^{21}$, Ferdinando D’Amico ${ }^{22}$, Meyno Den Dulk ${ }^{23}$, Barbara Elzbieta Dyrda $^{24}$, Virginia Festa ${ }^{25}$, Sara Gallina ${ }^{26}$, Rossella Grasso ${ }^{27}$, Jozef Hanzel $^{28}$, Jomni Mohamed Taieb ${ }^{29}$, Maria Antonia Lai ${ }^{30}$, Giovanni Latella $^{31}$, Daniele Lisi ${ }^{32}$, Lucia Lodi ${ }^{33}$, Stefania Marangi ${ }^{34}$, Alessandro Mardegan $^{35}$, Wojciech Marlicz ${ }^{36}$, Attilio Maurano ${ }^{37}$, Giuseppe Milazzo $^{38}$, Valentin Militaru ${ }^{39}$, Stefania Miraglia ${ }^{40}$, Fabio Monica ${ }^{41}$, Alex Moskalev ${ }^{42}$, Antonino Natale ${ }^{43}$, Christelle Nicolas ${ }^{24}$, Andrea Pancetti ${ }^{44}$, Antonio Penna ${ }^{45}$, Angelo Santo Pepe ${ }^{46}$, Marcello Pisano ${ }^{47}$, Stefano Pontone ${ }^{48}$, Marta Prati ${ }^{49}$, Antonio Prisco ${ }^{50}$, Letterio Rando ${ }^{51}$, Eduardo Rodriguez Hernandez ${ }^{52}$, Ottorino Rosati ${ }^{53}$, Giacomo Rossi ${ }^{54}$, Giovanni Rubis Passoni ${ }^{55}$, Valerio Papa ${ }^{56}$, Noel Salgado Nesme ${ }^{57}$, Luigi Schiffino $^{15}$, Dario Schillaci ${ }^{58}$, Giuseppe Selvaggi ${ }^{59}$, Fabrizio Taborchi ${ }^{60}$, Alessandro Tornar ${ }^{61}$, František Trebuna ${ }^{62}$, Ciro Triggiani ${ }^{63}$, Filippo Vasile Testai ${ }^{64}$, Roberto Vassallo ${ }^{64}$, Alessandra Violi1 ${ }^{4}$
1) Division of Gastroenterology, "Santa Caterina Novella” Hospital, Galatina (LE), Italy; 2) Division of Gastroenterology, "Federico Spaziani” Hospital, Frosinone, Italy; 3) Ambulatory of Digestive Endoscopy, "Endoscopia Digestiva LDA”, Coimbra, Portugal; 4) Division of Gastroenterology, IRCCS “San Donato", San Donato Milanese (MI), Italy; 5) Digestive Endoscopy Unit, "G. Martino" University Hospital, Messina, Italy; 6) Division of Emergency and General Surgery, “Umberto I” Hospital, Siracusa, Italy; 7) Digestive Endoscopy Unit, "Villa Verde" Home Care, Reggio Emilia, Italy; 8) Division of Gastroenterology and Digestive Endoscopy, "Augusto Murri" Hospital, Fermo, Italy; 9) Division of Gastroenterology, "Cà Foncello" Hospital, ULSS 9, Treviso - Italy; 10) Digestive Endoscopy Unit, A.O. "Papardo", Messina, Italy; 11) Digestive Endoscopy Unit, Monselice Hospital, Monselice (PD), Italy; 12) Gastroenterology Sevice, EPS “Hédi Chaker”, Sfax, Tunisie; 13) Territorial Gastroenterology Service, Azienda Sanitaria Locale Benevento, Benevento, Italy; 14) Department of Medicine and Surgery, Gastroenterology Unit, University of Parma, Parma, Italy; 15) Division of Digestive Surgical Endoscopy, “G.B. Grassi” Hospital, Ostia (Roma), Italy; 16) General Pratictioner and Gastroenterologist, Azienda Sanitaria Locale BAT, San Ferdinando di Puglia (BT), Italy; 17) Department of Gastroenterology, Acibadem City Clinic, Sofia,Bulgaria; 18) General Pratictioner and Gastroenterologist, Azienda Sanitaria Locale Roma E, Roma, Italy; 19) Ambulatory of Gastroenterology "S. Antonio" Home Care, Cagliari, Italy; 20) Department of Clinical and Experimental Medicine, University „Federico II”, Naples, Italy ; 21) Department of Gastroenterology and Hepatology, Institutul Clinic Fundeni, Bucharest, Romania; 22) Division of Gastroenterology, "Humanitas" University Hospital, Rozzano (MI), Italy; 23) Medical Centre de Veluwe, Apeldoorn, The Netherlands; 24) Division of Internal Medicine and Gastroenterology, "Cristo Re" Hospital, Rome, Italy; 25) Division of Gastroenterology, "S. Filippo Neri” Hospital, Rome, Italy; 26) Division of Gastroenterology, "Belcolle" Hospital, Viterbo, Italy; 27) Division of Internal medicine and Gastroenterology, "V. Cervello" Hospital, Palermo, Italy; 28) Division of Laparoscopic Surgery, "Zlaté Moravce” Hospital, Jelenec, Slovakia; 29) Service de Gastro-entérologie, Hôpital des FSI La Marsa, Tunis, Tunisia; 30) Digestive Endoscopy Unit, “Monferrato” University Hospital, Cagliari, Italy; 31) Division of Gastroenterology, "San Salvatore” Hospital, L'Aquila University, L’Aquila, Italy; 32) Territorial Gastroenterology Service, ASL Roma 2, Rome, Italy; 33) Division of Internal Medicine and Gastroenterology, Busto Arsizio Hospital, Busto Arsizio (VA), Italy; 34) Division of Gastroenterology, IRCCS "Saverio De Bellis", Castellana Grotte (BA), Italy; 35) IV Division of Surgery, "Cà Foncello" Hospital, ULSS 9, Treviso, Italy; 36) Department of Gastroenterology, Pomeranian Medical University, Szczecin, Poland; 37) Division of Digestive Endoscopy, "Curteri” Hospital, Mercato S. Severino (SA), Italy; 38) Division of Gastroenterology and Digestive Endoscopy, "S. Biagio" Hospital, Marsala (TP) - Italy; 39) 2nd Medical Department, Iuliu Hatieganu University of Medicine and Pharmacy Cluj-Napoca, Romania; 40) Division of Gastroenterology, AO "ARNAS Garibaldi”, Catania, Italy; 41) Division of Gastroenterology and Digestive Endoscopy, AOU "Ospedali Riuniti, Trieste, Italy; 42) Laboratory of Molecular Radiobiology and Gerontology Institute of Biology, Institute of Biology of Komi, Komi, Russia; 43) General Pratictioner and Gastroenterologist, Azienda Sanitaria Locale BAT, Bisceglie (BT), Italy; 44) Gastrointestinal Unit, Department of Clinical and Experimental Medicine, University of Pisa, Pisa, Italy; 45) Division of Gastroenterology, "S. Paolo" Hospital, Bari, Italy; 46) 
Division of Surgery, "S.S. Annunziata” Hospital, Taranto, Italy; 47) Division of Surgery, "S. Marcellino" Hospital, ASL 8, Muravera (CA), Italy; 48) First Division of General Surgery, Digestive Endoscopy Unit, “Umberto I" University Hospital, "Sapienza” University, Rome, Italy; 49) Division of Surgery "Santi Antonio e Margherita" Hospital, Tortona (ALI), Italy; 50) Division of Gastroenteroloscy, "T. Maresca" Hospital, Torre del Greco (NA), Italy; 51) Surgical Endoscopy Unit, “G. Martino" University Hospital, Messina, Italy; 52) 28Sección de Cirugía Gastrointestinal, Hospital Universitari del Mar, Barcelona, Spain; 53) Division of General Surgery, G and C "Mazzoni” Hospital, Ascoli Piceno, Italy; 54) Digestive Endoscopy Unit, Voghera Hospital, ASST, Voghera (PV), Italy; 55) Division of Digestive Endoscopy, "S. Carlo Borromeo" Hospital, Milano, Italy; 56) Division of General Surgery, "S. Carlo di Nancy" Hospital, Rome, Italy; 57) Instituto Nacional de Ciencias Médicas y Nutrición Salvador Zubirán, Ciudad de México, Mexico; 58) Division of Surgery, "Suor Cecilia Basarocco" Hospital, Niscemi (PA), Italy; 59) Service of Digestive Endoscopy, „Rocco Mazzarone” Territorial Hospital, Tricarico (MT), Italy; 60) Digestive Endoscopy Unit, A.O. “Santa Maria”, Terni, Italy; 61) 1st Division of Internal Medicine, "Felice Lotti" Hospital, Azienda USL Toscana Nord Ovest, Pontedera (FI), Italy; 62) Ambulatory of Gastroenterology, Košice, Slovakia; 63) Division of Internal Medicine "Francesco Lastaria" Hospital, Lucera (FG), Italy ; 64) Division of Gastroenterology, "Bucchieri - La Ferla” Hospital, Palermo, Italy.

\section{REFERENCES}

1. Tursi A. Diverticulosis today: unfashionable and still underresearched. Therap Adv Gastroenterol 2016;9:213-228. doi:10.1177/1756283X15621228

2. Tursi A, Papa A, Danese S. Review article: the pathophysiology and medical management of diverticulosis and diverticular disease of the colon. Aliment Pharmacol Ther 2015;42:664-684. doi:10.1111/apt.13322

3. Lohrmann C, Ghanem N, Pache G, Makowiec F, Kotter E, Langer M. CT in acute perforated sigmoid diverticulitis. Eur J Radiol 2005;56:78-83. doi:10.1016/j.ejrad.2005.03.003

4. Wasvary H, Turfah F, Kadrom O, Beauregard W. Same hospitalization resection for acute diverticulitis. Am Surg 1999;65:632-635.

5. Ambrosetti P, Becker C, Terrier F. Colonic diverticulitis: impact of imaging on surgical management - a prospective study of 542 patients Eur Radiol 2002;12:1145-1149. doi:10.1007/s00330-001-1143-y

6. Köhler L, Sauerland S, Neugebauer E. Diagnosis and treatment of diverticular disease: results of a consensus development conference.
The Scientific Committee of the European Association for Endoscopic Surgery. Surg Endosc 1999;13:430-436. doi:10.1007/s004649901007

7. Sheth AA, Longo W, Floch MH. Diverticular disease and diverticulitis. Am J Gastroenterol 2008;103:1550-1556.

8. Hansen O, Graupe F, Stock W. Prognostic factors in perforating diverticulitis of the large intestine. Chirurg 1998;69:443-449. doi:10.1007/s001040050436

9. Ghorai S, Ulbright TM, Rex DK. Endoscopic findings of diverticular inflammation in colonoscopy patients without clinical acute diverticulitis: prevalence and endoscopic spectrum. Am J Gastroenterol 2003;98:802-806.

10. Tursi A, Elisei W, Giorgetti GM, Aiello F, Brandimarte G. Inflammatory manifestations at colonoscopy in patients with colonic diverticular disease. Aliment Pharmacol Ther 2011;33:358-365. doi:10.1111/j.13652036.2010.04530.x

11. Tursi A, Brandimarte G, Di Mario F, et al. Development and validation of an endoscopic classification of diverticular disease of the colon: the DICA classification. Dig Dis 2015;33:68-76. doi:10.1159/000366039

12. Tursi A, Brandimarte G, Di Mario F, et al. Predictive value of the Diverticular Inflammation and Complication Assessment (DICA) endoscopic classification on the outcome of diverticular disease of the colon: An international study. United European Gastroenterol J 2016;4:604-613. doi:10.1177/2050640615617636

13. Tursi A, Brandimarte G, Di Mario F, et al. P.02.12 Predictive value of the "DICA" endoscopic classification on the outcome of diverticular disease of the colon: a 1-year analysis from the international, multicenter, prospective study”. Dig Liver Dis 2019;51(Suppl 2):e152-e153. doi:10.1016/S1590-8658(19)30297-X

14. Tursi A, Brandimarte G, di Mario F, et al. The "DICA" endoscopic classification for diverticular disease of the colon shows a significant interobserver agreement among community endoscopists. J Gastrointestin Liver Dis 2019;28:23-27. doi:10.15403/jgld.2014.1121.281.dic

15. Fleiss JL. Statistical methods for rates and proportions. 2nd ed. New York: John Wiley and Sons 1981:211-236.

16. Randolph JJ. Online Kappa Calculator, 2008. Accessed February 21, 2013. Available at: http://justusrandolph.net/kappa/

17. Rath HC, Timmer A, Kunkel C, et al. Comparison of interobserver agreement for different scoring systems for reflux esophagitis: Impact of level of experience. Gastrointest Endosc 2004;60:44-49. doi:10.1016/ s0016-5107(04)01289-1

18. Daperno $\mathrm{M}$, Comberlato $\mathrm{M}$, Bossa $\mathrm{F}$, et al. Inter-observer agreement in endoscopic scoring systems: preliminary report of an ongoing study from the Italian Group for Inflammatory Bowel Disease (IG-IBD). Dig Liver Dis 2014;46:969-973. doi:10.1016/j.dld.2014.07.010 